Pacific Journal of Mathematics

ANALYTIC FUNCTIONS WITH VALUES IN A FRECHET SPACE 


\section{ANALYTIC FUNCTIONS WITH VALUES \\ IN A FRECHET SPACE}

\section{ERRETT Bishop}

We wish to extend certain results in the theory of analytic functions of several complex variables to the case of analytic functions with values in a Frechet space $F$. To do this, we prove (Theorem 1 below) that such a function $\varphi$ has an expansion of the form

$$
\varphi=\sum_{n=1}^{\infty} P_{n} \circ \varphi,
$$

where $\left\{P_{n}\right\}$ is a sequence of continuous mutually annihilating projections on $F$ whose ranges are all one-dimensional subspaces of $F$. This representation reduces the study of $\varphi$, for many purposes, to the study of the functions $P_{n} \circ \varphi$, which are essentially scalar-valued analytic functions. We actually prove the stronger (and more useful) result that if $\left\{\varphi_{k}\right\}$ is a sequence of analytic functions with values in $F$ then a single sequence $\left\{P_{n}\right\}$ can be found to give an expansion $(*)$ for every $\varphi_{k}$. Expansions of vector-valued functions of a different type have been considered by Grothendick [6].

Theorem 1 is applied to generalize Theorem B of H. Cartan [3]. We consider a coherent analytic sheaf $S$ on a Stein manifold $M$ and introduce the notion of the vectorization $S_{F}$ of $S$ (relative to a given Frechet space $F$ ).

If 0 denotes the sheaf of locally-defined analytic functions and $0_{F}$ denotes the sheaf of locally-defined analytic functions with values in $F$, then $S_{F}$ is defined to be the tensor product $S \otimes 0_{F}$ of the 0 -modules $S$ and $0_{F}$. For the important case of a coherent analytic subsheaf $S$ of the sheaf $0^{k}$ of locally-defined $k$-tuples of analytic functions, $S_{F}$ turns out to be canonically isomorphic to the sheaf $S_{F}^{\prime}$ determined by assigning to each open set $U$ the module of all $k$-tuples $\left(f_{1}, \cdots, f_{k}\right)$ of analytic functions from $U$ to $F$ which have the property that for each $u$ in $F^{*}$ the $k$-tuple $\left(u \circ f_{1}, \cdots, u \circ f_{k}\right)$ is a cross-section of $S$ over $U$. For instance, if $S$ is the sheaf of all locally-defined analytic functions which vanish on a given analytic set $A$ then it is evident that $S_{F}^{\prime}$ is the sheaf of all locally-defined analytic functions with values in $F$ which vanish on $A$.

One of the main results, an extension of Theorem $B$ of [3], will be that the cohomology groups $H^{N}\left(M, S_{F}\right)$ vanish in all dimensions $N \geqq 1$, where $S_{F}$ is the vectorization of a coherent analytic sheaf $S$ on a Stein manifold $M$. Using this theorem and the isomorphism of $S_{F}$ to the sheaf $S_{F}^{\prime}$ defined above one could show, for instance, that the usual

Received January 15, 1962. This work was partially supported by the Sloan Foundation. 
sheaf-theoretic solutions to Cousin's problems carry over to the case of analytic functions with values in a Frechet space. Special cases were treated by totally different methods in [2], but the techniques of that paper seem to be inadequate to obtain general results.

The proofs are all Banach-space theoretic. That is, only Banach space theory is necessary to obtain the above extension of Theorem B and to prove the necessary facts about vectorizations. We begin with a theorem which is given without proof on p. 278 of Banach [1], who attributes it to H. Auerbach. A proof can be found in Taylor [7]. Since complex Banach spaces are considered here, we give the proof.

THEOREM (Auerbach). An n-dimensional Banach space $B$ has a basis of unit vectors whose dual basis also consists of unit vectors.

Proof. Choose a basis $\left(b^{1}, \cdots, b^{n}\right)$ of $B$ and for any $x$ in $B$ let $\left(x_{1}, \cdots, x_{n}\right)$ be the coordinates of $x$ relative to the chosen basis. Let $T$ be the set of all $n$-tuples $\left(x^{1}, \cdots, x^{n}\right)$ of unit vectors in $B$. For each $\left(x^{1}, \cdots, x^{n}\right)$ in $T$ let $\alpha\left(x^{1}, \cdots, x^{n}\right)$ be the absolute value of the determinant $\operatorname{det}\left(x_{j}^{i}\right)$. Thus $\alpha$ is a continuous function on the compact space T. Now $\alpha\left(x^{1}, \cdots, x^{n}\right) \neq 0$ if and only if $\left(x^{1}, \cdots, x^{n}\right)$ is a basis. Thus $\alpha$ attains its maximum for $T$ at some point $\left(y^{1}, \cdots, y^{n}\right)$ in $T$ which is a basis of unit vectors. Let $\left(u^{1}, \cdots, u^{n}\right)$ be the dual basis in $B^{*}$. Now $\left\|u^{i}\right\| \geqq 1$ because $\left\langle y^{i}, u^{i}\right\rangle=1$. Assume $\left\|u^{i}\right\|>1$ for some $i$. Thus there exists $t$ in $B$ with $\|t\|=1$ and $\left.\left\langle t, u^{i}\right\rangle=c\right\rangle 1$. Thus $\left\langle t-c y^{i}, u^{i}\right\rangle=$ 0 , so that $t-c y^{i}$ is a linear combination of the vectors of the basis $\left(y^{1}, \cdots, y^{n}\right)$ other than $y^{i}$. If we let $\left(z^{1}, \cdots, z^{n}\right)$ be the basis $\left(y^{1}, \cdots, y^{n}\right)$ with $y^{i}$ replaced by $t$ it follows that $\alpha\left(z^{1}, \cdots, z^{n}\right)=c \alpha\left(y^{1}, \cdots, y^{n}\right)$. Since the basis $\left(z^{1}, \cdots, z^{n}\right)$ consists of unit vectors this contradicts the choice of $\left(y^{1}, \cdots, y^{n}\right)$. Thus $\left\|u^{i}\right\|=1$ for all $i$, and the theorem is proved.

Corollary. If $B_{0}$ is a finite-dimensional subspace of dimension $n$ of a Banach space $B$ there exist $n$ mutually annihilating projections (idempotent continuous linear operators) on $B$, each of norm 1 , whose ranges are one-dimensional subspaces of $B_{0}$ and whose sum is a projection of $B$ onto $B_{0}$ of norm at most $n$.

Proof. Let $\left(y^{1}, \cdots, y^{n}\right)$ be a basis of unit vectors of $B_{0}$ such that the dual basis $\left(u^{1}, \cdots, u^{n}\right)$ of $B_{0}^{*}$ also consists of unit vectors. Let $v^{i}$ be an extension of $u^{i}$ to a linear functional on $B$ of norm 1. The operators $P_{1}, \cdots, P_{n}$ on $B$ defined by

$$
P_{i} x=\left\langle x, v^{i}\right\rangle y^{i}
$$

are the desired projections.

We recall that a Frechet space is a locally convex topological linear 
space $F$ which admits a countable family $\left\{\|\|_{k}\right\}$ of continuous seminorms such that a basis for the neighborhoods of 0 in $F$ is given by the sets

$$
\left\{x \in F:\|x\|_{k}<1\right\} .
$$

If \|\| is any continuous semi-norm on $F$ it follows that for some $k$ $\|x\| \leqq\|x\|_{k}$ for all $x$ in $F$. If necessary it may be assumed that $\left\{\|\|_{k}\right\}$ is a monotonely nondecreasing sequence of semi-norms, in which case we shall call it a defining sequence of semi-norms for $F$.

Lemma 1. Let $F$ be a Frechet space with a defining sequence $\left\{\|\|_{k}\right\}$ of semi-norms. Let $\left\{a_{n}\right\}$ be a sequence of vectors in $F,\left\{\delta_{k}\right\} a$ sequence of nonnegative real numbers, and $\left\{k_{j}\right\}$ a strictly increasing sequence of positive integers. Then there exists a sequence $\left\{P_{n}\right\}$ of mutually annihilating continuous projections on $F$, whose ranges are subspaces of $F$ of dimensions at most 1 , and a sequence $\left\{\varepsilon_{k}\right\}$, with $0<\varepsilon_{k}<\delta_{k}$ for all $k$, with the following properties. For each positive integer $j$ the operator

$$
Q_{j}=\sum_{n=1}^{k_{j}} P_{n}
$$

is a projection on the subspace $B_{j}$ of $F$ spanned by the vectors $a_{1}, \cdots, a_{k_{j}}$. For each positive integer $n$ the sum

$$
\|a\|_{0}=\sum_{k=1}^{\infty} \varepsilon_{k}\|a\|_{k}
$$

is finite for $a=a_{n}$. For each positive integer $j$ and all $n \leqq k_{j}$ we have $\left\|P_{n}\right\|_{0} \leqq\left(1+k_{1}^{2}\right) \cdots\left(1+k_{j}^{2}\right)$, where

$$
\left\|P_{n}\right\|_{0}=\sup \left\{\left\|P_{n} b\right\|_{0}: b \in F, \quad\|b\|_{0}=1\right\} \text {. }
$$

Proof. We may assume the $\delta_{k}$ to be so small that $\sum_{k=1}^{\infty} \delta_{k}\left\|a_{n}\right\|_{k}<\infty$ for all $n$. By induction we construct a sequence $\left\{P_{n}\right\}$ of mutually annihilating continuous projections, a sequence $\left\{\varepsilon_{k}\right\}$ of positive real numbers, and an increasing sequence $\left\{N_{j}\right\}$ of positive integers such that

(a) $0<\varepsilon_{k}<\delta_{k}$,

(b) For each $j$ the operator $Q_{j}$ is a projection onto $B_{j}$,

(c) $\left\|P_{n}\right\|^{j}<\left(1+k_{1}^{2}\right) \cdots\left(1+k_{i}^{2}\right)$ for $1 \leqq n \leqq k_{i}$ and all $i \leqq j$.

We explain what is meant by (c). First of all, \|\|$^{j}$ is the continuous semi-norm on $F$ defined by

$$
\|b\|^{j}=\sum_{k=1}^{N_{j}} \varepsilon_{k}\|b\|_{k} .
$$

Secondly, $\left\|P_{n}\right\|^{j}$ is defined by

$$
\left\|P_{n}\right\|^{\jmath}=\sup \left\{\left\|P_{n} b\right\|^{\jmath}:\|b\|^{j}=1\right\} \text {. }
$$


Assuming that $P_{1}, \cdots, P_{k,}$ and $N_{1} \cdots, N_{j}$, and $\varepsilon_{1}, \cdots, \varepsilon_{N_{j}}$ have been found with the relevant properties, we show how to continue to the next stage $j+1$. First choose $N_{j+1}>N_{j}$ so large that \|\|$_{N_{j+1}}$ is a norm (and not merely a semi-norm) on $B_{j+1}$. Choose then $\varepsilon_{i}, N_{j}<i \leqq$ $N_{j+1}$, so small that $0<\varepsilon_{i}<\delta_{i}$ and $\left\|P_{n}\right\|^{j+1}<\left(1+k_{1}^{2}\right) \cdots\left(1+k_{i}^{2}\right)$ for $n \leqq k_{j}$ and all $i \leqq j$. To see that this can be done, notice that because \|\|$_{N}$, is a norm on $B$, there exists $r>0$ so that $r\|a\|^{j}>\|a\|_{m}$ for all $a$ in $B_{j}$ and all $m \leqq N_{j+1}$. Thus

$$
\left\|P_{n}\right\|^{j+1} \leqq \sup \left\{\left\|P_{n} b\right\|^{j+1}:\|b\|^{j}=1\right\} \leqq\left(1+\sum_{m=N_{j}+1}^{N} \varepsilon_{m}\right)\left\|P_{n}\right\|^{j} .
$$

Now use (c).

Now let $Q_{j}^{\prime}$ be the restriction of $Q_{j}$ to $B_{j+1}$ and let $I_{j+1}$ be the identity operator on $B_{j+1}$. Thus $I_{j+1}-Q_{j}^{\prime}$ is a projection of $B_{j+1}$ onto a subspace $S_{j+1}$. Clearly $B_{j}$ and $S_{j+1}$ are complementary subspaces of $B_{j+1}$, so that $\operatorname{dim} S_{j+1} \leqq k_{j+1}-k_{j}$. By the above corollary there exists a projection $E_{j+1}$ with $\left\|E_{j+1}\right\|^{j+1} \leqq k_{j+1}$ of $F$ onto $B_{j+1}$. Also by the above corollary there exist mutually annihilating projections $R_{n}, k_{j}<n \leqq$ $k_{j+1}$, of $S_{j+1}$ onto subspaces of dimensions at most 1 such that $\left\|R_{n}\right\|^{j+1} \leqq 1$ for all $n$ and such that $\Sigma R_{n}$ is the identity projection of $S_{j+1}$ onto itself. For $k_{j}<n \leqq k_{j+1}$ we define

$$
P_{n}=R_{n}\left(I_{j+1}-Q_{j}^{\prime}\right) E_{j+1} .
$$

Thus the $P_{n}$ are mutually annihilating projections for $1 \leqq n \leqq k_{j+1}$. Also $Q_{j+1}$ is a projection onto $B_{j+1}$. Finally for $k_{j}<n \leqq k_{j+1}$ we have

$$
\begin{aligned}
\left\|P_{n}\right\|^{j+1} & \leqq\left\|R_{n}\right\|^{j+1}\left\|I_{j+1}-Q_{j}^{\prime}\right\|^{j+1}\left\|E_{j+1}\right\|^{j+1} \\
& \leqq\left(1+\sum_{n=1}^{k_{j}}\left\|P_{n}\right\|^{j+1}\right) k_{j+1} \\
& <\left[1+k_{j}\left(1+k_{1}^{2}\right) \cdots\left(1+k_{j}^{2}\right)\right] k_{j+1} \\
& \leqq\left(1+k_{1}^{2}\right) \cdots\left(1+k_{j+1}^{2}\right) .
\end{aligned}
$$

The same is true for $n \leqq k_{j}$, by the above construction. Thus the construction has been continued another step. By induction it follows that sequences $\left\{P_{n}\right\},\left\{N_{j}\right\}$, and $\left\{\varepsilon_{k}\right\}$ can be chosen satisfying properties (a), (b), and (c). It is immediate that the sequences $\left\{P_{n}\right\}$ and $\left\{\varepsilon_{k}\right\}$ satisfy the requirements of the lemma.

Lemma 2. Let $\left\{a_{n}\right\}$ be a sequence of elements of a Frechet space $F,\left\{\|\|_{k}\right\}$ a defining sequence of semi-norms on $F$, and $\left\{\delta_{k}\right\}$ a sequence of positive real numbers. Then there exist a sequence $\left\{\varepsilon_{k}\right\}$ of positive real numbers and a sequence $\left\{P_{n}\right\}$ of mutually annihilating projections on $F$ whose ranges are subspaces of $F$ of dimensions at most 1 having the following properties. 
(i) $0<\varepsilon_{k}<\delta_{k}$ for all $k$,

(ii) For $a=a_{n}$ the norm $\|a\|_{0}=\sum_{k=1}^{\infty} \varepsilon_{k}\|a\|_{k}$ is finite for all n,

(iii) $R_{m} a_{n}=a_{n}$ for all positive integers $m$ and $n$ with $m \geqq 2 n$, where $R_{m}=\sum_{j=1}^{m} P_{j}$,

(vi) For all $t>1$ and $\varepsilon>0$ the sum $\sum_{n=1}^{\infty}\left\|P_{n}\right\|_{0} t^{-n^{\varepsilon}}$ converges, where $\left\|P_{n}\right\|_{0}$ is defined as above.

Proof. Define the sequence $\left\{k_{j}\right\}$ by $k_{j}=2^{j}$. Choose the sequences $\left\{P_{n}\right\}$ and $\left\{\varepsilon_{k}\right\}$ as in lemma 1. Clearly (i) and (ii) are satisfied. Now for each positive integer $n$ there is a positive integer $j$ with $2^{j-1} \leqq n<2^{j}$. It follows that $a_{n} \in B_{j}$. Thus $R_{2^{j}} a_{n}=Q_{j} a_{n}=a_{n}$, so that $R_{m} a_{n}=a_{n}$ for all $m \geqq 2^{j}$ and therefore for all $m \geqq 2 n$. This proves (iii).

Now for each $n$ choose $j$ with $2^{j-1} \leqq n<2^{j}$. Thus

$$
\begin{aligned}
\left\|P_{n}\right\|_{0} & \leqq\left(1+k_{j}^{2}\right)^{j}=\left(1+2^{2 j}\right)^{j} \\
& \leqq\left(5 n^{2}\right)^{j} \leqq\left(5 n^{2}\right)^{\infty}
\end{aligned}
$$

where $\alpha=1+\log _{2} n$. From this it follows from elementary calculus that (iv) holds, thereby proving the lemma.

LEMMA 3. Let

$$
\sum_{n_{1} \geqq 0, \cdots, n_{\alpha} \geqq 0} a_{i}\left(n_{1}, \cdots, n_{\alpha}\right) z_{1}^{n_{1}} \cdots z_{\alpha}^{n_{\alpha}}
$$

where $\alpha=\alpha_{i}$ and $1 \leqq i<\infty$, be a sequence of formal power series with coefficients in a Frechet space $F$. Let $\left\{\delta_{k}\right\}$ be a sequence of positive real numbers. Then there exists a sequence $\left\{\varepsilon_{k}\right\}$ with $0<\varepsilon_{k}<\delta_{k}$ for all $k$ and $a$ sequence $\left\{P_{n}\right\}$ of mutually annihilating continuous projections of $F$ onto subspaces of dimensions at most 1 such that

(a) $R_{m} a_{i}\left(n_{1}, \cdots, n_{\alpha}\right)=a_{i}\left(n_{1}, \cdots, n_{\alpha}\right)$ whenever $m \geqq 2^{i+2} n^{\alpha}$, where $\alpha=\alpha_{i}, n=n_{1}+\cdots+n_{\alpha}$, and $R_{m}=\sum_{j=1}^{m} P_{j}$,

(b) $P_{m} a_{i}\left(n_{1}, \cdots, n_{\alpha}\right)=0$ whenever $m>2^{i+2} n^{\alpha}$,

(c) $\sum_{n=1}^{\infty}\left\|P_{n}\right\|_{0} t^{-n^{\varepsilon}}<\infty$ for all $t>1$ and $\varepsilon>0$, where \|\|$_{0}$ is defined as above.

Proof. For each $i$ order the coefficients $a_{i}\left(n_{1}, \cdots, n_{\alpha}\right)$ into a sequence $\left\{\alpha_{i}^{k}\right\}_{k=1}^{\infty}$ according to the size of $n$. We now define a sequence $\left\{a_{k}\right\}$ of elements of $F$ which is an ordering of the totality of the $a_{i}\left(n_{1}, \cdots, n_{\alpha}\right)$. For $k$ given let $2^{i}$ be the largest power of 2 dividing $k$ and let $j=$ $1 / 2\left(k 2^{-i}+1\right)$. Let $a_{k}=\alpha_{i}^{j}$. Now choose the sequences $\left\{\varepsilon_{k}\right\}$ and $\left\{P_{n}\right\}$ as in Lemma 2. Clearly (c) holds. Since (b) is a consequence of (a) we need only check (a). To this end consider a fixed $a_{i}\left(n_{1}, \cdots, n_{\alpha}\right)$. Now there exists $j \leqq n^{\alpha}$ with $a_{i}\left(n_{1}, \cdots, n_{\alpha}\right)=\alpha_{i}^{j}$. In turn $\alpha_{i}^{j}=a_{k}$ for some $k \leqq 2^{i+1} n^{\alpha}$. By (iii) of Lemma 2 it follows that $R_{m} a_{k}=a_{k}$ for $m \geqq 2 k$ and therefore for $m \geqq 2^{i+2} n^{\alpha}$, as was to be proved. 
We are now prepared to prove a series representation for analytic functions with values in a Frechet space which will be the principal tool in subsequent proofs.

THeorem 1. Let $F$ be a Frechet space and let $\left\{M_{i}\right\}$ be a sequence of complex analytic manifolds. For each $i$ let $\varphi_{i}$ be an analytic function on $M_{i}$ with values in $F$. Then there exists a sequence of vectors $\left\{b_{n}\right\}$ in $F$ and a sequence $\left\{P_{n}\right\}$ of continuous mutually annihilating projections of $F$ onto one-dimensional subspaces having the following properties. For each $i$ the series $\sum_{n=1}^{\infty} P_{n} \circ \varphi_{i}$ converges to $\varphi_{i}$ on $M_{i}$. For each $n$ we have $P_{n} b_{n}=b_{n}$, so that $P_{n} \circ \varphi_{i}=\varphi_{i}^{n} b_{n}$, for some analytic function $\varphi_{i}^{n}$ on $M_{i}$. For each $i$ the series $\sum_{n=1}^{\infty} \varphi_{i}^{n}$ converges absolutely and uniformly on all compact subsets of $M_{i}$. For each continuous semi-norm \|\| on $F$ the sequence $\left\{\left\|b_{n}\right\|\right\}$ is bounded.

Proof. For each $i$ let $\operatorname{dim} M_{i}=\alpha=\alpha_{i}$, so that $M_{i}$ is coverable by a countable family of analytic homeomorphs $\Gamma$ of the unit polycylinder

$$
U^{\alpha}=\left\{z=\left(z_{1}, \cdots, z_{\alpha}\right):\left|z_{j}\right|<1,1 \leqq j \leqq \alpha\right\} .
$$

Thus in the proof of the theorem we may replace the sequence $\left\{M_{i}\right\}$ by the totality of all such $\Gamma$. There is therefore no loss of generality in assuming that each $M_{i}$ is a polycylinder $U^{\alpha}$ of dimension $\alpha=\alpha_{i}$. Let $\left\{\|\|_{k}\right\}$ be a defining sequence of semi-norms on $F$. Now for each $i$ the analytic function $\varphi_{i}$ has a power series expansion

$$
\varphi_{i}=\sum_{n_{1} \geqq 0, \cdots, n_{\alpha} \geqq 0} a_{i}\left(n_{1}, \cdots, n_{\alpha}\right) z_{1}^{n_{1}} \cdots z_{\alpha}^{n_{\alpha}}
$$

on the polycylinder $M_{i}=U^{\alpha}$. This expansion converges absolutely and uniformly on each compact subset of $M_{i}$ in each semi-norm \|\|$_{k}$. By the diagonal process there therefore exist constants $\delta_{k}>0$ such that the power series for each $\varphi_{i}$ converges absolutely and uniformly on each compact subset of $M_{i}$ in the norm $\sum_{k=1}^{\infty} \delta_{k}\|\|_{k}$, so that in particular this norm is finite for each coefficient $a_{i}\left(n_{1}, \cdots, n_{\alpha}\right)$. Now choose the sequences $\left\{\varepsilon_{k}\right\}$ and $\left\{P_{n}\right\}$ as in Lemma 3 relative to the power series expansions of the $\varphi_{i}$ and to the $\delta_{k}$ just obtained. Thus the power series for $\varphi_{i}$ converges absolutely and uniformly on compact subsets of $M_{i}$ in the norm \|\|$_{0}$ defined above. If some of the projections $P_{n}$ are zero, these may be omitted from the sequence. Thus for each $n$ there is a vector $b_{n}$ in $F$ with $\left\|b_{n}\right\|_{0}=1$ spanning the range of $P_{n}$. To show that the sequences $\left\{P_{n}\right\}$ and $\left\{b_{n}\right\}$ have the desired properties, consider a fixed compact subset $T$ of a fixed $M_{i}$. For each $n$ write

$$
\gamma_{n}=\sum_{n_{1}+\cdots+n_{\alpha}=n} \max \left\{\left\|a_{i}\left(n_{1}, \cdots, n_{\alpha}\right) z_{1}^{n_{1}} \cdots z_{\alpha}^{n_{\alpha}}\right\|_{0}: z \in T\right\} .
$$


By the usual convergence criteria we see that there exist $r>1$ and $c>0$ such that $r^{n} \gamma_{n}<c$ for all $n$.

If $j$ is any positive integer let $k$ be the largest integer such that $2^{i+2} k^{\alpha}<j$. Thus for each $z$ in $T$ we have

$$
\begin{aligned}
& \left\|P_{j} \varphi_{i}(z)\right\|_{0} \\
& \quad=\left\|P_{j} \sum_{n_{1}+\cdots+n_{\alpha} \geqq k} a_{i}\left(n_{1}, \cdots, n_{\alpha}\right) z_{1}^{n_{1}} \cdots z_{\alpha}^{n_{\alpha}}\right\|_{0} \\
& \quad \leqq\left\|P_{j}\right\|_{0} \sum_{n \geqq k} \gamma_{n} \leqq c\left\|P_{j}\right\|_{0} \sum_{n \geqq k} r^{-n} \\
& \quad=c\left(1-r^{-1}\right)^{-1}\left\|P_{j}\right\|_{0} r^{-k} .
\end{aligned}
$$

Thus

$$
\begin{aligned}
\Delta & =\max \left\{\sum_{j=1}^{\infty}\left\|P_{j} \varphi_{i}(z)\right\|_{0}: z \in T\right\} \\
& \leqq c\left(1-r^{-1}\right)^{-1} \sum_{j=1}^{\infty} r^{-k}\left\|P_{j}\right\|_{0} .
\end{aligned}
$$

Now by the definition of $k$ we see that $k$ is the integral part of $\left(j 2^{-i-2}\right)^{1 / \alpha}$, so that $k \geqq j^{1 / 2 \alpha}$ for all $j$ sufficiently large. Thus $\Delta$ is finite if the sum $\sum_{j=1}^{\infty} r^{-j^{8}}\left\|P_{j}\right\|_{0}$ converges, where $\varepsilon=(2 a)^{-1}$. By the choice of the sequence $\left\{P_{j}\right\}$ this series converges so that $\Delta$ is finite. Now since $\left\|b_{n}\right\|_{0}=1$,

$$
\max \left\{\left|\varphi_{i}^{n}(z)\right|: z \in T\right\}=\max \left\{\left\|P_{n} \varphi_{i}(z)\right\|_{0}: z \in T\right\} .
$$

Therefore the series $\sum_{n=1}^{\infty} \varphi_{i}^{n}(z)$ converges absolutely and uniformly on $T$. If \|\| is a continuous semi-norm on $F$ then \|\|$\leqq K\|\|_{0}$ for some $K>0$, so that $\left\{\left\|b_{n}\right\|\right\}$ is bounded by $K$. Finally, we must show that $\sum_{n=1}^{\infty} P_{n} \circ \varphi_{i}$ actually converges to $\varphi_{i}$ (and not to something else). To see this, note by (a) and (b) of Lemma 3 that $R_{m} \circ \varphi_{i}$ and $\varphi_{i}$ have power series expansions in the coordinates $z_{1}, \cdots, z_{\infty}$ which agree up to terms of total order $n$, whenever $m \geqq 2^{i+2} n^{\alpha}$. This completes the proof of Theorem 1.

Before giving the definition of the vectorization of an analytic sheaf, we indicate the terminology to be used, following Godement [5]. A presheaf $S$ on a topological space $X$ assigns to each open $U \subset X$ a set $S(U)$ and to each open set $V \subset U \subset X$ a map $r_{V U}: S(U) \rightarrow S(V)$ satisfying $r_{W V} \circ r_{V U}=r_{W U}$ for $W \subset V \subset U$. In particular the same terminology will be used if $S$ is a sheaf, that is, a presheaf satisfying axioms (F1) and (F2) on page 109 of [5]. To any presheaf $S$ is canonically associated a sheaf $S^{\prime}$, and each element $f$ in $S(U)$ gives rise to a unique element in $S^{\prime}(U)$ which will also be denoted by $f$. If $X$ is a complex analytic manifold a sheaf $S$ on $X$ is called analytic if it is a module over the sheaf 0 of locally defined analytic functions, that is, if for each $U$ the set $S(U)$ is an $0(U)$-module, and if the usual com- 
mutation relations between module multiplication and the restriction maps $S(U) \rightarrow S(V)$ and $0(U) \rightarrow 0(V)$ hold.

DEFINITION 1. Let $S$ be an analytic sheaf on a complex analytic manifold $M$ and let $F$ be a Frechet space. Let 0 be the sheaf of locally-defined analytic functions on $M$ and let $0_{F}$ be the sheaf of locallydefined analytic functions on $M$ with values in $F$, where by definition a continuous function $f$ from an open set $U \subset M$ to $F$ is called analytic if $u \circ f$ is analytic for all $u$ in $F^{*}$. Clearly $0_{F}$ is an 0 -module, i.e., an analytic sheaf. The vectorization $S_{F}$ of $S$ (relative to $F$ ) is defined to be the sheaf $S \otimes 0_{F}$, the tensor product of the 0 -modules $S$ and $0_{F}$. This is defined in [5] as the sheaf determined by the presheaf data

$$
U \rightarrow S(U) \otimes 0_{F}(U),
$$

where $S(U)$ and $0_{F}(U)$ are considered as $0(U)$-modules, together with the obvious restriction maps.

Note that if $T$ is a continuous linear operator from a Frechet space $F$ into a Frechet space $G$ then the natural homomorphism $T_{0}$ of $0_{F}$ into $0_{\theta}$ induced by $T$ gives rise to a homomorphism $T^{\prime}=1 \otimes T_{0}$ of $S_{F}$ into $S_{G}$. In particular, if $u$ is an element of $F^{*}$ (and so a continuous linear operator from $F$ into $C$ ) then $u$ induces a homomorphism of $S_{F}$ into $S_{\sigma}$. But $S_{o}$ is canonically isomorphic to $S$, in virtue of the canonical isomorphism between the $0(U)$-modules $S(U) \otimes 0(U)$ and $S(U)$. (See [5] p. 8.) If we identify $S_{o}$ with $S$ it follows that each $u$ in $F^{*}$ induces a homomorphism $u^{\prime}$ of $S_{F}$ onto $S$.

DEFINITION 2. If $S$ is an analytic subsheaf of the Cartesian product $0^{n}$ we define

$$
S_{F}^{\prime}(U)=\left\{f \in\left(0_{F}(U)\right)^{n}: u \circ f \in S(U) \text { for all } u \text { in } F^{*}\right\} .
$$

Clearly $S_{F}^{\prime}$ so defined is an analytic subsheaf of the Cartesian product $\left(0_{F}\right)^{n}$.

THEOREM 2. If $S$ is a coherent analytic subsheaf of $0^{n}$ then to each $p$ in $U \subset M$ and each $f$ in $S_{F}^{\prime}(U)$ there exists a neighborhood $V$ of $p$, functions $H_{1}, \cdots, H_{k}$ in $S(V)$ and functions $G_{1}, \cdots, G_{k}$ in $0_{F}(V)$ such that

$$
r_{V U} f=\sum_{m=1}^{k} G_{m} H_{m}
$$

Proof. Since $S$ is coherent, there exists a neighborhood $V_{0} \subset U$ of $p$ and functions $H_{1}, \cdots, H_{k}$ in $S\left(V_{0}\right)$ which generate $S$ at each point of $V_{0}$. We may assume that $\bar{V}_{0}$ is a compact subset of $U$. Let $V_{0} \supset V_{1} \supset V_{2} \supset \cdots$ 
be a basis for the neighborhoods of $p$. Let $\Omega$ be the subset of $S\left(V_{0}\right)$ consisting of all elements in $S\left(V_{0}\right)$ which as elements of $\left(0\left(V_{0}\right)\right)^{n}$ are bounded on $V_{0}$. Thus to each $h$ in $\Omega$ there exists $G=\left(G_{1}, \cdots, G_{k}\right)$ in $\left(0\left(V_{i}\right)\right)^{k}$ for some $i$ such that the restriction of $h$ to $V_{i}$ has the form

$$
h=\sum_{i=1}^{k} G_{i} H_{i} .
$$

By choosing $i$ large enough we may assume that

$$
\|G\|_{i}=\sup \left\{\left|G_{j}(q)\right|: q \in V_{i}, 1 \leqq j \leqq k\right\}
$$

is finite. Thus if for each pair $(i, N)$ of positive integers we let $\Omega_{i N}$ be the family of all $h$ in $\Omega$ such that $G$ can be chosen in $\left(0\left(V_{i}\right)\right)^{k}$ with

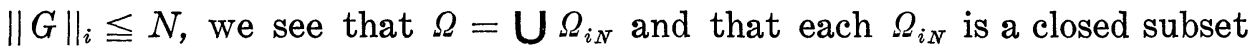
of $\Omega$, where $\Omega$ has the norm defined by

$$
\|h\|_{0}=\sup \left\{\left|h_{i}(q)\right|: 1 \leqq i \leqq n, q \in V_{0}\right\}
$$

for each $h=\left(h_{1}, \cdots, h_{n}\right) \in \Omega \subset\left(0\left(V_{0}\right)\right)^{n}$. By the Baire category theorem there exists $(i, N)$ such that $\Omega_{i N}$ has a nonvoid interior. From this it follows as usual that there exists a constant $K>0$ such that for each $h$ in $\Omega$ there exists $G$ in $\left(0\left(V_{i}\right)\right)^{k}$ as above with $\|G\|_{i} \leqq K\|h\|_{0}$. Now consider $f$ as in the statement of the theorem, so that $f \in S_{F}^{\prime}(U) \subset\left(0_{F}(U)\right)^{n}$. By Theorem 1 there exists a sequence of vectors $\left\{b_{j}\right\}$ in $F$ which is bounded in each continuous semi-norm on $F$ and a sequence $\left\{P_{j}\right\}$ of continuous projections on $F$ having one-dimensional ranges such that $\sum_{j=1}^{\infty} P_{j} \circ f$ converges uniformly to $f$ on all compact subsets of $U$ and such that for each $j$ we have $P_{j} \circ f=f_{j} b_{j}$ with $f_{j} \in(0(U))^{n}$, where $\sum_{j=1}^{\infty}\left|f_{j}\right|$ converges uniformly on all compact subsets of $U$. Thus $\sum_{j=1}^{\infty}\left\|f_{j}\right\|_{0}$ is finite, since $\bar{V}_{0} \subset U$.

Now for each $j$ there exists $u$ in $F^{*}$ with $\left\langle b_{j}, u\right\rangle=1$. Thus

$$
f_{j}=u \circ\left(f_{j} b_{j}\right)=u \circ\left(P_{j} \circ f\right)=\left(u \circ P_{j}\right) \circ f
$$

is in $S(U)$ because $f \in S_{F}^{\prime}(U)$ and $u \circ P_{j} \in F^{*}$. Thus $f_{j} \in S(U)$ for all $j$. By the above for each $j$ there exists $G^{j}=\left(G_{1}^{j}, \cdots, G_{k}^{j}\right)$ in $\left(0\left(V_{i}\right)\right)^{k}$ such that on $V_{i}$ we have

$$
f_{j}=\sum_{m=1}^{k} G_{m}^{j} H_{m},
$$

with $\left\|G^{j}\right\|_{i} \leqq K\left\|f_{j}\right\|_{0}$. It follows that the series $\sum_{j=1}^{\infty} G^{j} b_{j}$ converges uniformly and absolutely on $V_{i}$ in each continuous semi-norm on $F$. Thus the sum of this series is an element $G=\left(G_{1}, \cdots, G_{k}\right)$ in $\left(0_{F}\left(V_{i}\right)\right)^{k}$. Thus in the topology of uniform and absolute convergence on compact subsets of $V_{i}$ in each continuous semi-norm on $F$ we have 


$$
\begin{aligned}
f & =\lim _{t \rightarrow \infty} \sum_{j=1}^{t} f_{j} b_{j} \\
& =\lim _{t \rightarrow \infty} \sum_{j=1}^{t} \sum_{m=1}^{k} G_{m}^{j} H_{m} b_{j} \\
& =\sum_{m=1}^{k}\left(\lim _{t \rightarrow \infty} \sum_{j=1}^{t} G_{m}^{j} b_{j}\right) H_{m} \\
& =\sum_{m=1}^{k} G_{m} H_{m},
\end{aligned}
$$

as was to be proved.

The following consequence of Theorem 2 will be useful later.

LEMMA 4. If the element $f$ of $S_{F}(U)$ has the property that $u^{\prime} f$ is the zero element of $S(U)$ for all $u$ in $F^{*}$ then $f=0$.

Proof. By taking a covering of $U$ by small open sets we reduce to the case in which $f$ has a representation

$$
f=\sum_{i=1}^{k} h_{i} \otimes g_{i}
$$

with $h_{i}$ in $S(U)$ and $g_{i}$ in $0_{F}(U)$. Let $R$ be the sheaf on $U$ of relations of $h_{1}, \cdots, h_{k}$. Thus for each $u$ in $F^{*}$ we see that

$$
\begin{aligned}
0 & =u^{\prime} f=\sum_{i=1}^{k} h_{i} \otimes\left\langle g_{i}, u\right\rangle \\
& =\sum_{i=1}^{k}\left\langle g_{i}, u\right\rangle h_{i} .
\end{aligned}
$$

Thus by Definition 2 we see that $g=\left(g_{1}, \cdots, g_{k}\right) \in R_{F}^{\prime}(U)$. By Theorem 2 it follows that each $p$ in $U$ has a neighborhood $V \subset U$ such that there exist $H_{1}, \cdots, H_{t}$ in $R(V)$ and $G_{1}, \cdots, G_{t}$ in $0_{F}(V)$ with

$$
r_{V U} g=\sum_{j=1}^{t} G_{j} H_{j} .
$$

Thus for each $i$ with $1 \leqq i \leqq k$ we have

$$
r_{V U} g_{i}=\sum_{j=1}^{t} G_{j} H_{j}^{i},
$$

where $H_{j}=\left(H_{j}^{1}, \cdots, H_{j}^{k}\right)$. Therefore on $V$ we have

$$
\begin{aligned}
f & =\sum_{i=1}^{k} h_{i} \otimes g_{i}=\sum_{i=1}^{k} h_{i} \otimes\left(\sum_{j=1}^{t} G_{j} H_{j}^{i}\right) \\
& =\sum_{i=1}^{k}\left(\sum_{j=1}^{t} h_{i} \otimes\left(G_{i} H_{j}^{i}\right)\right) \\
& =\sum_{j=1}^{t}\left(\sum_{i=1}^{k} H_{j}^{i} h_{i}\right) \otimes G_{j}=0
\end{aligned}
$$


since $H_{j} \in R(V)$ for all $j$. This proves Lemma 4 .

We next give an important characterization of $S_{F}$ in case $S$ is a coherent analytic subsheaf of $0^{n}$ for some positive integer $n$.

TheOREM 3. Let $M$ be a Stein manifold and $S$ a coherent analytic subsheaf of $0^{n}$. Let $F$ be a Frechet space. For each open $U \subset M$ there is a mapping $\tau(U)$ from $S(U) \otimes 0_{F}(U)$ into $\left(0_{F}(U)\right)^{n}$ which for each $h=\left(h_{1}, \cdots, h_{n}\right)$ in $S(U)$ and $g$ in $0_{F}(U)$ maps $h \otimes g$ onto $g h=\left(g h_{1}, \cdots, g h_{n}\right)$ in $\left(0_{F}(U)\right)^{n}$. For each such $g$ and $h$ the image gh of $h \otimes g$ actually lies in the subset $S_{F}^{\prime}(U)$ of $\left(0_{F}(U)\right)^{n}$. The family of such mappings $\tau(U)$ induces an isomorphism $\tau$ of the sheaf $S_{F}$ (which was defined above to be the sheaf determined by the presheaf data $U \rightarrow S(U) \otimes 0_{F}(U)$ ) onto the sheaf $S_{F}^{\prime}$. Thus $S_{F}^{\prime}$ and $S_{F}$ are isomorphic.

Proof. Clearly the map of the Cartesian product $S(U) \times 0_{F}(U)$ into $\left(0_{F}(U)\right)^{n}$ defined by $(h, g) \rightarrow g h$ induces a group homomorphism of $\left(S(U), 0_{F}(U)\right)$-the free abelian group generated by the elements of the Cartesian product $S(U) \times 0_{F}(U)$-into $\left(0_{F}(U)\right)^{n}$. It is trivial to check that $N\left(S(U), 0_{F}(U)\right)$ : belongs to the kernel of this map, where $N\left(S(U), 0_{F}(U)\right)$ is defined as in [5] p. 8 to be the subgroup of $\left(S(U), 0_{F}(U)\right)$ generated by elements of the forms

$$
\begin{array}{ll}
\text { (i) } & \left(x_{1}+x_{2}, y\right)-\left(x_{1}, y\right)-\left(x_{2}, y\right) \\
\text { (ii) } & \left(x, y_{1}+y_{2}\right)-\left(x, y_{1}\right)-\left(x, y_{2}\right) \\
\text { (iii) } & (a x, y)-(x, a y)
\end{array}
$$

where $x, x_{1}$, and $x_{2}$ are in $S(U), y, y_{1}$, and $y_{2}$ are in $0_{F}(U)$, and $a \in 0(U)$. Thus this map induces a homomorphism $\tau(U)$ of the quotient $\left(S(U), 0_{F}(U)\right) / N\left(S(U), 0_{F}(U)\right)=S(U) \otimes 0_{F}(U)$ into $\left(0_{F}(U)\right)^{n}$. It is trivial to check that $\tau(U)$ is an $0(U)$-homomorphism. Now with $g$ and $h$ as above and $u$ in $F^{*}$ we have

$$
u \circ \tau(U)(h \otimes g)=u \circ(g h)=(u \circ g) h \in S(U) .
$$

Thus $\tau(U)(h \otimes g) \in S_{F}^{\prime}(U)$. It follows that the range of $\tau(U)$ is a subset of $S_{F}^{\prime}(U)$. It is now clear that the family of mappings $\tau(U)$ induces an 0 -homomorphism $\tau$ of $S_{F}$ into $S_{F}^{\prime}$. To show that $\tau$ is one-to-one we must prove

(a) If $\tau(U)\left(\sum_{i=1}^{N} h_{i} \otimes g_{i}\right)=0$ then each $p$ in $U$ has a neighborhood $V$ such that $r_{V U}\left(\sum_{i=1}^{N} h_{i} \otimes g_{i}\right)=0$.

To show that $\tau$ is onto we must prove

(b) If $f \in S_{F}^{\prime}(U)$ then each $\mathrm{p}$ in $U$ has a neighborhood $V$ such that $r_{V U} f=\tau(V)\left(\sum_{i=1}^{N} h_{i} \otimes g_{i}\right)$ for some elements $h_{i}$ in $S(V)$ and $g_{i}$ in $0_{F}(V)$. We first prove (a). If we let $R$ be the sheaf of relations on $U$ of $h_{1}, \cdots, h_{N}$ by the coherence of $R$ there exists a neighborhood $V_{0}$ of $p$ and elements $r_{1}=\left(r_{1}^{1}, \cdots, r_{1}^{N}\right), \cdots, r_{n}=\left(r_{n}^{1}, \cdots, r_{n}^{N}\right)$ of $R\left(V_{0}\right)$ which 
generate $R$ at each point of $V_{0}$. Now

$$
\sum_{i=1}^{N} g_{i} h_{i}=\tau(U)\left(\sum_{i=1}^{N} h_{i} \otimes g_{i}\right)=0 .
$$

Thus for each $u$ in $F^{*}$ we have

$$
\sum_{i=1}^{N}\left(u \circ g_{i}\right) h_{i}=0
$$

so that $\left(u \circ g_{1}, \cdots, u \circ g_{N}\right) \in R(U)$ for all $u$ in $F^{*}$. By definition this means that $\left(g_{1}, \cdots, g_{N}\right) \in R_{F}^{\prime}(U)$. Therefore by Theorem 2 we see that there exists a neighborhood $V$ of $p$ and $G=\left(G_{1}, \cdots, G_{n}\right)$ in $\left(0_{F}(V)\right)^{n_{n}}$ such that $\left(g_{1}, \cdots, g_{N}\right)=G_{1} r_{1}+\cdots+G_{n} r_{n}$. Thus on $V$ we have

$$
\begin{aligned}
\sum_{i=1}^{N} h_{i} \otimes g_{i} & =\sum_{i=1}^{N} h_{i} \otimes\left(\sum_{j=1}^{n} G_{j} r_{j}^{i}\right) \\
& =\sum_{j=1}^{n}\left(\sum_{i=1}^{N}\left(r_{j}^{i} h_{i}\right)\right) \otimes G_{j}=0
\end{aligned}
$$

since $r_{j} \in R(V)$ for each $j$. This proves (a).

To prove (b) notice by Theorem 2 that there exists a neighborhood $V$ of $p$, elements $h_{1}, \cdots, h_{N}$ in $S(V)$, and elements $g_{1}, \cdots, g_{N}$ in $0_{F}(V)$, such that on $V$ we have

$$
f=\sum_{i=1}^{N} g_{i} h_{i}=\tau(V)\left(\sum_{i=1}^{N} h_{i} \otimes g_{i}\right) .
$$

This completes the proof of Theorem 3.

We state for future reference a version of a theorem of Banach, first giving a definition.

Definition 3. If $\left\{g_{n}\right\}$ is a sequence of vectors in a Frechet space $F_{\infty}$ the series $\sum_{n=1}^{\infty} g_{n}$ is called absolutely convergent if the series $\sum_{n=1}^{\infty}\left\|g_{n}\right\|$ converges for each continuous semi-norm \|\| on $F$.

Notice that a continuous linear transformation from a Frechet space $F$ to a Frechet space $G$ takes absolutely convergent sequences into absolutely convergent sequences.

Lemma 5. Let $\sigma$ be a continuous linear map of a Frechet space $F$ onto a Frechet space $G$. Let $\left\{g_{i}\right\}$ be an absolutely convergent sequence from $G$. Then there exists an absolutely convergent sequence $\left\{f_{i}\right\}$ in $F$ such that $\sigma\left(f_{i}\right)=g_{i}$ for all $i$.

Proof. Let $\left\{\|\|_{k}\right\}$ be a defining sequence of semi-norms on $F$. Since the map $\sigma$ is continuous, we see ([1] p. 40) that for each $k$ the set $\sigma\left\{f:\|f\|_{k} \leqq 1\right\}$ contains a neighborhood $\left\{g:\|g\|_{k}^{\prime} \leqq 1\right\}$ of 0 in $G$, where \|\|$_{k}^{\prime}$ is some continuous semi-norm on $G$. Thus for each $g$ in 
$G$ and each $k$ there exists $f$ in $F$ with $\sigma(f)=g$ and $\|f\|_{k} \leqq\|g\|_{k}^{\prime}$. Now for each $k$ choose $j=j(k)$ such that

$$
\sum_{n=j}^{\infty}\left\|g_{n}\right\|_{k}^{\prime}<2^{-k}
$$

so that

$$
\sum_{k=1}^{\infty} \sum_{n=j(k)}^{\infty}\left\|g_{n}\right\|_{k}^{\prime}<\infty
$$

We may assume that $j(1)<j(2)<\cdots$. For each $n$ with $j(k) \leqq n<j(k+1)$ choose $f_{n}$ in $F$ with $\sigma\left(f_{n}\right)=g_{n}$ and $\left\|f_{n}\right\|_{k} \leqq\left\|g_{n}\right\|_{k}^{\prime}$. If for each $n$ we let $k(n)$ be the smallest value of $k$ for which $n<j(k+1)$, it follows that

$$
\sum_{n=1}^{\infty}\left\|f_{n}\right\|_{k(n)}<\infty .
$$

Since for each $t$ we have $\left\|f_{n}\right\|_{t} \leqq\left\|f_{n}\right\|_{k}$ for all $k \geqq t$ it follows that

$$
\sum_{n=1}^{\infty}\left\|f_{n}\right\|_{t}
$$

is finite for all $t$. This proves the lemma.

THEOREM 4. If $S$ is a coherent analytic sheaf on a Stein manifold $M$ and if $F$ is a Frechet space then $H^{N}\left(M, S_{F}\right)=0$ for all $N \geqq 1$.

Proof. Let $f$ be an element of $H^{N}\left(M, S_{F}\right)$. Consider a locally finite covering $\left\{U_{i}\right\}$ of $M$ by holomorphically convex open sets $U_{i}$, so fine that $f$ is represented by an element of $H^{N}\left(\left\{U_{i}\right\}, S_{F}\right)$. For each finite sequence $K=\left(i_{1}, \cdots, i_{k}\right)$ of positive integers let $U_{K}=U_{i_{1}} \cap \cdots \cap U_{i_{k}}$. The element $f$ of $H^{N}\left(M, S_{F}\right)$ can be considered to belong to $H^{N}\left(\left\{U_{i}\right\}, S_{F}\right)$ and therefore can be represented by a cocycle $f=\left\{f_{I}\right\}$ of $Z^{N}\left(\left\{U_{i}\right\}, S_{F}\right)$. Here $I$ is any sequence of $N+1$ positive integers, and, for each $I, f_{I}$ is an element of $S_{F}\left(U_{I}\right)$. Also $\delta f=0$, where $\delta$ is the coboundary operator from $C^{N}\left(\left\{U_{i}\right\}, S_{F}\right)$ into $C^{N+1}\left(\left\{U_{i}\right\}, S_{F}\right)$ and $Z^{N}\left(\left\{U_{i}\right\}, S_{F}\right)$ is the kernel of $\delta$. By choosing the covering $\left\{U_{i}\right\}$ fine enough we may assume that for each $K$ there exist elements $h_{1 K}, \cdots, h_{\alpha_{K}}$, with $\alpha$ depending on $K$, in $S\left(U_{K}\right)$ which generate $S$ at each point of $U_{K}$. This implies ([3], expose XVIII, p. 9) that every $h$ in $S\left(U_{K}\right)$ has a representation of the form $h=\sum_{i=1}^{\alpha} g_{i} h_{i K}$, with $g_{i} \in 0\left(U_{K}\right)$. We may also choose the covering $\left\{U_{i}\right\}$ so fine that, for each $I, f_{I}$ can be represented in the form

$$
f_{I}=\sum_{i=1}^{\infty} h_{i I} \otimes g_{i I}
$$

with $h_{i I}$ as above and with $g_{i I}$ in $0_{F}\left(U_{I}\right)$. 
By Theorem 1 there exists a sequence $\left\{P_{n}\right\}$ of continuous mutually annihilating projections on $F$ whose ranges are one dimensional and a sequence $\left\{b_{n}\right\}$ of vectors in $F$ bounded in each continuous semi-norm on $F$ having the following properties. For each $I$ and $i$ the series $\sum_{n=1}^{\infty} P_{n} \circ g_{i r}$ converges to $g_{i I}$ on $U_{I}$. For each $I$ and $i$ we have $P_{n} \circ g_{i I}=g_{i I}^{n} b_{n}$, where $g_{i I}^{n} \in 0\left(U_{I}\right)$. For each $I$ and $i$ the series $\sum_{n=1}^{\infty} g_{i I}^{n}$ converges absolutely in the Frechet space $0\left(U_{I}\right)$. Now since for each $n$ the projection $P_{n}$ induces a homomorphism of the sheaf $S_{F}$ onto itself, the element $\left\{P_{n} f_{I}\right\}$ of $C^{N}\left(\left\{U_{i}\right\}, S_{F}\right)$ is in $Z^{N}\left(\left\{U_{i}\right\}, S_{F}\right)$. Also

$$
\begin{aligned}
P_{n} f_{I} & =\sum_{i=1}^{\infty} h_{i I} \otimes P_{n} g_{i I} \\
& =\sum_{i=1}^{\alpha} h_{i I} \otimes g_{i I}^{n} h_{n}=\left(\sum_{\imath=1}^{\infty} g_{i I}^{n} h_{i I}\right) \otimes b_{n} .
\end{aligned}
$$

If for each $n$ and $I$ we let $f_{I}^{n}$ be the element $\sum_{i=1}^{\infty} g_{i I}^{n} h_{i I}$ of $S\left(U_{I}\right)$ it follows that for each $n$ the element $f^{n}=\left\{f_{I}^{n}\right\}^{i=1}$ of $C^{N}\left(\left\{U_{i}\right\}, S\right)$ belongs to $Z^{N}\left(\left\{U_{i}\right\}, S\right)$. It is also clear that $f^{n} b_{n}=P_{n} f$.

Now there exists a natural Frechet space topology on each $S(U)$, described in [4], expose XVII. This topology has the property that for each $h$ in $S(U)$ the map $g \rightarrow g h$ of $0(U)$ into $S(U)$ is continuous. We therefore see that for each $I$ the series

$$
\sum_{n=1}^{\infty} f_{I}^{n}=\sum_{n=1}^{\infty}\left(\sum_{i=1}^{\infty} g_{i I}^{n} h_{i I}\right)
$$

converges absolutely in $S\left(U_{I}\right)$ because for each $I$ and $i$ the series $\sum_{n=1}^{\infty} g_{n I}^{n}$ converges absolutely in $0\left(U_{I}\right)$. Now the space $C^{N}\left(\left\{U_{i}\right\}, S\right)$ is the Cartesian product of the Frechet spaces $S\left(U_{I}\right)$, and therefore possesses a Frechet space structure. Moreover $Z^{N}\left(\left\{U_{i}\right\}, S\right)$ is closed in $C^{N}\left(\left\{U_{i}\right\}, S\right)$ and is therefore also a Frechet space. Since for each $I$ the series $\sum_{n=1}^{\infty} f_{I}^{n}$ converges absolutely in $S\left(U_{I}\right)$ it follows that $\sum_{n=1}^{\infty} f^{n}$ converges absolutely in $Z^{N}\left(\left\{U_{i}\right\}, S\right)$. By Theorem B of [3] and Leray's theorem (see [5] p. 213) we see that the coboundary map $\delta$ of the Frechet space $C^{N-1}\left(\left\{U_{i}\right\}, S\right)$ into $Z^{N}\left(\left\{U_{i}\right\}, S\right)$ is onto. From [4] we also see that $\delta$ is continuous.

Let $J$ stand for an arbitrary $N$-tuple of positive integers. Thus for each $J$, by the above, there is a continuous homomorphism.

$$
\tau_{J}:\left(G_{1}, \cdots, G_{\alpha}\right) \rightarrow \sum_{i=1}^{\alpha} G_{i} h_{i J}
$$

of the Frechet space $\left(0\left(U_{J}\right)\right)^{\alpha}$ onto the Frechet space $S\left(U_{J}\right)$. These maps induce a continuous homomorphism $\tau$ of the Frechet space $\Phi$ onto the Frechet space $C^{N-1}\left(\left\{U_{i}\right\}, S\right)$, where $\Phi$ is defined to be the product $\Pi_{J}\left(0\left(U_{J}\right)\right)^{\alpha}$, with $\alpha$ depending as above on $J$, of the Frechet spaces $\left(0\left(U_{J}\right)\right)^{\alpha}$. Thus 


$$
\sigma=\delta \circ \tau
$$

is a continuous homomorphism of $\Phi$ onto $Z^{N}\left(\left\{U_{i}\right\}, S\right)$. Since $\sum_{n=1}^{\infty} f^{n}$ converges absolutely in $Z^{N}\left(\left\{U_{i}\right\}, S\right)$ it follows from Lemma 5 that there exists an absolutely convergent sequence $\left\{G^{n}\right\}$ in $\Phi$ with $\sigma\left(G^{n}\right)=f^{n}$ for all $n$. For each $n$ write $G^{n}=\left\{G_{J}^{n}\right\}$, where

$$
G_{J}^{n}=\left(G_{1 J}^{n}, \cdots, G_{\alpha J}^{n}\right) \in\left(0\left(U_{J}\right)\right)^{\infty} .
$$

Thus for each $J$ we see that the series $\sum_{n=1}^{\infty} G_{J}^{n}$ converges absolutely and uniformly on every compact subset of $U_{J}$, so that the series $\sum_{n=1}^{\infty} G_{J}^{n} b_{n}$ converges absolutely in $\left(0_{F}\left(U_{J}\right)\right)^{\alpha}$ to an element

$$
G_{J}=\left(G_{1 J}, \cdots, G_{\alpha J}\right)
$$

in $\left(0_{F}\left(U_{J}\right)\right)^{\alpha}$. Thus for each $i$ and $J$ we have $G_{i J}=\sum_{n=1}^{\infty} G_{i J}^{n} b_{n}$.

For each $J$ let $e_{J}$ be the element

$$
e_{J}=\sum_{i=1}^{\infty} h_{i J} \otimes G_{i J}
$$

of $S_{F}\left(U_{J}\right)$. Thus $e=\left\{e_{J}\right) \in C^{N-1}\left(\left\{U_{i}\right\}, S_{F}\right)$. We shall finish the proof by showing that $\delta e=f$. To this end it is sufficient by Lemma 4 to show $u^{\prime}(\delta e)=u^{\prime}(f)$ for all $u$ in $F^{*}$. We compute:

$$
\begin{aligned}
u^{\prime}\left(e_{J}\right) & =\sum_{i=1}^{\infty}\left\langle G_{i J}, u\right\rangle h_{i J} \\
& =\sum_{i=1}^{\infty}\left\langle\sum_{n=1}^{\infty} G_{i J}^{n} b_{n}, u\right\rangle h_{i J} \\
& =\sum_{n=1}^{\infty}\left(\sum_{i=1}^{\infty} G_{i J}^{n} h_{i J}\right)\left\langle b_{n}, u\right\rangle \\
& =\sum_{n=1}^{\infty}\left(\tau_{J}\left(G_{J}^{n}\right)\right)\left\langle b_{n}, u\right\rangle
\end{aligned}
$$

absolutely in $S\left(U_{J}\right)$. Thus

$$
u^{\prime}(e)=\sum_{n=1}^{\infty}\left(\tau\left(G^{n}\right)\right)\left\langle b_{n}, u\right\rangle
$$

absolutely in $C^{N-1}\left(\left\{U_{i}\right\}, S\right)$. Thus

$$
\begin{aligned}
u^{\prime}(\delta e) & =\delta\left(u^{\prime}(e)\right)=\sum_{n=1}^{\infty}(\delta \circ \tau)\left(G^{n}\right)\left\langle b_{n}, u\right\rangle \\
& =\sum_{n=1}^{\infty} \sigma\left(G^{n}\right)\left\langle b_{n}, u\right\rangle=\sum_{n=1}^{\infty} f^{n}\left\langle b_{n}, u\right\rangle .
\end{aligned}
$$

Also for each $I$ we have

$$
u^{\prime}\left(f_{I}\right)=\sum_{i=1}^{\alpha}\left\langle g_{i I}, u\right\rangle h_{i I}
$$




$$
\begin{aligned}
& =\sum_{i=1}^{\infty}\left\langle\sum_{n=1}^{\infty} g_{i I}^{n} b_{n}, u\right\rangle h_{i I} \\
& =\sum_{n=1}^{\infty}\left(\sum_{i=1}^{\infty} g_{i I}^{n} h_{i I}\right)\left\langle b_{n}, u\right\rangle=\sum_{n=1}^{\infty} f_{I}^{n}\left\langle b_{n}, u\right\rangle .
\end{aligned}
$$

Therefore $u^{\prime}(f)=\sum_{n=1}^{\infty} f^{n}\left\langle b_{n}, u\right\rangle$. It follows that $u^{\prime}(f)=u^{\prime}(\delta e)$ for all $u$ in $F^{*}$, so that $f=\delta e$. This completes the proof of Theorem 4.

\section{REFERENCES}

1. S. Banach, Theorie des operations lineaires, Warsaw, 1932.

2. E. Bishop, Some global problems in the theory of functions of several complex variables Amer. J. of Math., 83 (1961), 479-498.

3. H. Cartan, Seminaire Ecole Normale Superieure, 1951-1952.

4. - Seminaire Ecole Normale Superieure, 1952-1953.

5. R. Godement, Theorie des faisceaux, Paris, 1958.

6. A. Grothendieck, Sur certains espoces de fonctions holomorphes. II., Jour. für die reine und angewandte Math., 192 (1953), 77-95.

7. A. E. Taylor, A geometric theorem and its applications to biorthogonal systems, Bull. Amer. Math. Soc., 53 (1947), 614-616.

INSTITUTE FOR ADVANCED STUDY

Princeton, NeW Jersey 


\section{PACIFIC JOURNAL OF MATHEMATICS}

\section{EDITORS}

\author{
Ralph S. Phillips \\ Stanford University \\ Stanford, California \\ M. G. Arsove \\ University of Washington \\ Seattle 5 , Washington
}

\author{
A. L. Whiteman \\ University of Southern California \\ Los Angeles 7, California \\ Lowell J. Paige \\ University of California \\ Los Angeles 24, California
}
E. F. BECKENBACH
D. DERRY
ASSOCIATE EDITORS
T. M. CHERRY
M. OHTSUKA
H. L. ROYDEN
E. G. STRAUS
E. SPANIER
F. WOLF

\section{SUPPORTING INSTITUTIONS}

\author{
UNIVERSITY OF BRITISH COLUMBIA \\ CALIFORNIA INSTITUTE OF TECHNOLOGY \\ UNIVERSITY OF CALIFORNIA \\ MONTANA STATE UNIVERSITY \\ UNIVERSITY OF NEVADA \\ NEW MEXICO STATE UNIVERSITY \\ OREGON STATE UNIVERSITY \\ UNIVERSITY OF OREGON \\ OSAKA UNIVERSITY \\ UNIVERSITY OF SOUTHERN CALIFORNIA
}

\author{
STANFORD UNIVERSITY \\ UNIVERSITY OF TOKYO \\ UNIVERSITY OF UTAH \\ WASHINGTON STATE UNIVERSITY \\ UNIVERSITY OF WASHINGTON \\ AMERICAN MATHEMATICAL SOCIETY \\ CALIFORNIA RESEARCH CORPORATION \\ SPACE TECHNOLOGY LABORATORIES \\ NAVAL ORDNANCE TEST STATION
}

Mathematical papers intended for publication in the Pacific Journal of Mathematıcs should be typewritten (double spaced), and the author should keep a complete copy. Manuscripts may be sent to any one of the four editors. All other communications to the editors should be addressed to the managing editor, L. J. Paige at the University of California, Los Angeles 24, California.

50 reprints per author of each article are furnished free of charge; additional copies may be obtained at cost in multiples of 50 .

The Pacific Journal of Mathematics is published quarterly, in March, June, September, and December. Effective with Volume 13 the price per volume (4 numbers) is $\$ 18.00$; single issues, $\$ 5.00$. Special price for current issues to individual faculty members of supporting institutions and to individual members of the American Mathematical Society: $\$ 8.00$ per volume; single issues $\$ 2.50$. Back numbers are available.

Subscriptions, orders for back numbers, and changes of address should be sent to Pacific Journal of Mathematics, 103 Highland Boulevard, Berkeley 8, California.

Printed at Kokusai Bunken Insatsusha (International Academic Printing Co., Ltd.), No. 6, 2-chome, Fujimi-cho, Chiyoda-ku, Tokyo, Japan.

PUBLISHED BY PACIFIC JOURNAL OF MATHEMATICS, A NON-PROFIT CORPORATION

The Supporting Institutions listed above contribute to the cost of publication of this Journal, but they are not owners or publishers and have no responsibility for its content or policies. 


\section{Pacific Journal of Mathematics}

\section{Vol. 12 , No. 4 \\ April, 1962}

Tsuyoshi Andô, On fundamental properties of a Banach space with a cone ..... . 1163

Sterling K. Berberian, A note on hyponormal operators ................ 1171

Errett Albert Bishop, Analytic functions with values in a Frechet space . . . . . . . 1177

(Sherman) Elwood Bohn, Equicontinuity of solutions of a quasi-linear equation ............................................ 1193

Andrew Michael Bruckner and E. Ostrow, Some function classes related to the class of convex functions . . . . . . . . . . . . . . . . . . . . . . . . 1203

J. H. Curtiss, Limits and bounds for divided differences on a Jordan curve in the complex domain . . ................................. 1217

P. H. Doyle, III and John Gilbert Hocking, Dimensional invertibility . . . . . . . . 1235

David G. Feingold and Richard Steven Varga, Block diagonally dominant matrices and generalizations of the Gerschgorin circle theorem ................. 1241

Leonard Dubois Fountain and Lloyd Kenneth Jackson, A generalized solution of the boundary value problem for $y^{\prime \prime}=f\left(x, y, y^{\prime}\right) \ldots \ldots \ldots \ldots \ldots \ldots \ldots \ldots \ldots$

Robert William Gilmer, Jr., Rings in which semi-primary ideals are primary. . . . . 1273

Ruth Goodman, K-polar polynomials .......................... 1277

Israel Halperin and Maria Wonenburger, On the additivity of lattice completeness ........................................... 1289

Robert Winship Heath, Arc-wise connectedness in semi-metric spaces . . . . . . . . 1301

Isidore Heller and Alan Jerome Hoffman, On unimodular matrices . . . . . . . . . . . 1321

Robert G. Heyneman, Duality in general ergodic theory . . . . . . . . . . . . . . . 1329

Charles Ray Hobby, Abelian subgroups of p-groups . . . . . . . . . . . . . . . 1343

Kenneth Myron Hoffman and Hugo Rossi, The minimum boundary for an analytic

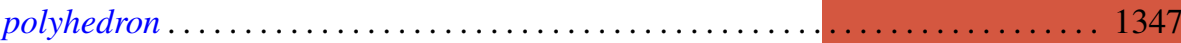

Adam Koranyi, The Bergman kernel function for tubes over convex cones ........ 1355

Pesi Rustom Masani and Jack Max Robertson, The time-domain analysis of a continuous parameter weakly stationary stochastic proces.

William Schumacher Massey, Non-existence of almost-complex structures on

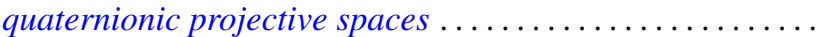

Deane Montgomery and Chung-Tao Yang, A theorem on the action of $\mathrm{SO}(3) \ldots . .1385$

Ronald John Nunke, A note on Abelian group extensions . . . . . . . . . . . . . 1401

Carl Mark Pearcy, A complete set of unitary invariants for operators generating finite $W^{*}$-algebras of type $I$

Edward C. Posner, Integral closure of rings of solutions of linear differential equations.

Duane Sather, Asymptotics. III. Stationary phase for two parameters with an application to Bessel functions.

J. Śladkowska, Bounds of analytic functions of two complex variables in domains

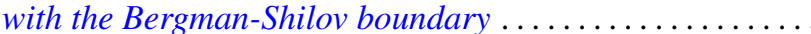

Joseph Gail Stampfli, Hyponormal operators .

George Gustave Weill, Some extremal properties of linear combinations of kernels

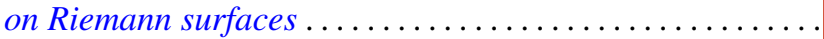

